American Medical Journal 2 (2): 79-86, 2011

ISSN 1949-0070

(C) 2011 Science Publications

\title{
Emotion Regulation in Childhood with Cancer (Acute Lymphocytic Leukemia)
}

\author{
${ }^{1}$ Siti Yuyun Rahayu Fitri, ${ }^{2}$ Ediasri T. Atmodiwirjo and ${ }^{2}$ Winarini Wilman \\ ${ }^{1}$ Department of Pediatric, Faculty of Nursing, Universities Padjadjaran, Bandung Indonesia \\ ${ }^{2}$ Department of Developmental Psychology, Faculty of Psychology, \\ Universities Indonesia, Jakarta, Indonesia
}

\begin{abstract}
Problem statement: Emotion regulation in children with Leukemia actively undergoing a chemotherapy program has distinctive forms of regulation in the research on different cultures. Furthermore, the capability of children to regulate their emotions during a dynamic process involving frequent hospitalization and painful treatment is of increasing interest to researchers. This must also take into account how the role of culture can influence emotion regulation. There is limited research about emotion regulation in sick children, especially children with Acute Lymphocytic Leukemia (ALL) in Indonesia. Thus in this study we aimed to explore emotion regulation in children with ALL during ongoing chemotherapy. Approach: A descriptive qualitative approach was chosen to carry out this study. The participants were six persons, consisting of four children aged 10-13 years old and two persons aged 20-21 years old. Emotion regulation according to Gross's theory comprises of situation selection, situation modification, attention deployment, cognitive change and response modulation. The emotion regulation was observed during various phases of the participants' chemotherapy treatment. These were the Induction, Consolidation and Maintenance Phases. Results: Children with leukemia have negative emotions, especially during the initial diagnosis, such as feeling sad, scared, angry, embarrassed and bored. These are experienced in various situations namely diagnosing reinforcement, chemotherapy preparation and the chemotherapy period. In managing their emotions, they develop emotion regulation in both interpersonal and interpersonal ways. An emotion regulation of focused response-is used more in the initial phase when children are diagnosed to have leukemia and antecedent-focused emotion regulation is seen in external parties. After undergoing chemotherapy for 1-3 months, the children are able to feel antecedent-focused emotion regulation in an interpersonal way and the emotion regulation of response focus decreases. Conclusion/Recommendations: Children with their capacity for development and supported by an external stimulus are capable of sorting particular responses in undertaking adaptive response alternatives. These responses can be used for various kinds of condition.
\end{abstract}

Key words: Emotion regulation, antecedent-focused, response focus, Acute Lymphocytic Leukemia (ALL), attacking organs, medical treatment, neural system, qualitative approach, antecedent focus, attentional deployment

\section{INTRODUCTION}

Acute Lymphositic Leucemia (ALL), a type of cancer that mostly attacks children, has good prognosis for living productively in the ensuing period (Kreitler and Arush, 2004). Today the number of children with ALL is increasing. As a result the treatment for it is also increasing, so that the number of deaths can be reduced. In addition, nowadays the population of children with cancer who can survivive is also increasing. Kreitler and Arush (2004) observes that the main focus in psycho-oncology is the life quality of the children with cancer, as well as the long term effects of cancer and its cure (through medical treatment).

ALL is a deasease with unpleasant physical symptoms, such as joint pains,weakness and other disorders attacking organs. Furthermore, medication for this type of cancer, especially chemotheraphy and radiation, causes physical discomfort, such as pains, nausea, vomit, hair loss through baldness (alopecia), severe sprue and loss of appetite. The physical effects will also be interelated with psychological aspects. This

Corresponding Author: Siti Yuyun Rahayu Fitri, Department of Pediatric, Faculty of Nursing, Universities Padjadjaran, Bandung Indonesia 
is because physical discomfort will create various feelings in the children, especially negative feelings and negative emotions.

Up till now, various research has mentioned more about the effects of ALL that tend to be negative in terms of the psychological aspects. In the early diagnosis, generally, people have the perception that cancer is the real killer, causing severe pain, disability and physical defects (Sarafino, 2005). Even among patients experiencing remission from medical treatment who adapt well in the first month toand first year, distress concerning medical treatment can be even more frightening than the disease itself (Andersen et al., 2000). Other research shows that a patient with cancer has difficulty in adapting to psychosocial post treatment, including post traumatic stress, depression, behavioural problems and deterioration in quality of life (Scwartz and Drotar, 2006; Kupst et al., 1995; Kazak, 1994; Speechley et al., 2006).

Children who experience psychological distress upon treatment frequently have low academic achievements and this makes it hard for them to get a job (Zeltzer et al., 1997; Zebrack et al., 2002). According to Coniglio (1995), children with LLA who get medical treatment risk problems in learning. These include include low IQ scores and deficits in Mathematics, attention and memory spans. These occur during medication phases and after, as the effect from one medicine in chemotheraphy (methorexate) that is given by way of intrathecal (through the spinal cord). Chen et al. (2000) suggests that children's memory about pain in cancer treatment plays an important role in distress and they experience this during the repetetition of the stress.

This is different from the results of Mahajan's Research in Kreitler and Arush (2004). These showed that more children have great ability in living with cancer experiences and are able to develop adequate psychosocial strength during their post treatment. Oppenheim, in Kreitler and Arush (2004), states that children's experiences in living with cancer vary and cannot be generalized. This depends on the children themselves, their families, their life condition, their way of thinking and reacting, their disease, their country's culture and how their medical team determines the treatment. Nevertheless, all children with cancer, whether they experience positive or negative effects during their medical treatment, will certainly experience various emotions. This is because emotions are feelings or affect that occur when someone is in a condition or experiencing an important interaction, especially in terms of their welfare.

Because of their disease and its unpleasant treatment, the early reactions of the children diagnosed as having cancer in certain phases show emotional reactions, especially negative emotions. According to Cole et al. (2004) emotion is the fastest reaction that appears in individuals when they are facing various situations. Generally, negative emotions cause more psychological problems. The appearance of the psychological problems has something to do with the role of individual's emotion regulation (Philippot and Feldman, 2004). Whether emotions that appear to be a big physiological and physichological problem or even affect well-being has something to do with the role of emotion regulation. Philippot and Feldman (2004) states that emotion regulation, both the one related to emotional experience control and management as well as emotional expression, are important aspects of daily functions. Failure to regulate emotions has negative consequences, at the individual or inter-individual levels. Sapolsky in Gross (2006) says that emotion regulation decreases patogen activation from stress response caused by psychological factors. It also increases behavioral response to psychological stressors selected adaptively by individuals. Emotion regulation can change a patient's perception towards their health. Furthermore, Middendorp et al. (2005) declared that the style of emotion regulation that makes the neural system relaxed can reduce complaints relating to the health aspects.

Furthermore, Middendorp et al. (2005) research showed that the style of the emotion regulation whereby the neural system became relaxed can reduce health complaints. The cultural values of individuals also colours action taken including emotion regulation.

The importance of development and culture is reflected in the cultural comparisons in the emotional representation and emotion regulation in children. At the age of 6 , for example, Nepali children are very different from American children. For example, in the way they sense their interpersonal conflict evaluation (when they feel shy or guilty) and their trust as to whether negative emotion should be expressed or not (Cole et al., 2002; Cole and Tamang, 1998). This kind of research shows that evaluation related to emotion in children is influenced by culture through socialization processes started at home. In cultures that emphasize collectivism, someone's emotional expressions or emotion regulation will consider and think more about public opinion.

Emotion regulation in children with Leukemia actively undergoing a chemotherapy program has distinctive forms of regulation according to the research on different cultures. Furthermore, the capability of children to regulate emotion during treatment that causes children to be hospitalized frequently and experience pain has interested researchers. 
Furthermore, development and the role of culture also appear to influence forms of emotion regulation.

There is limited research about emotion regulation in sick children in Indonesia, especially children with ALL. We therefore aim to explore emotion regulation among children with ALL undergoing chemotherapy in Indonesia using a qualitative approach.

Review of literature: Emotion regulation refers to the heterogeneous set of processes by which emotions are themselves regulated. The following five points represent five families of emotion regulation processes: situation selection, situation modification, attentional deployment, cognitive change and response modulation (Gross, 2006).

Situation selection: This is behavior socializing or creating distance with people or certain situations based on expected emotion effect.

Modification situation: This is modifying an existing situation, which cannot be selected so that the emotion effect is unavoidable. This is due to external and physical environmental modification in the form of the appearance of other individuals and action from an individual, or environment physical modification.

Attention deployment: This is behavior that shows the way someone pays attention in a situation to control emotion and can be considered as the internal version of situation selection. The main strategy in this group is concentration and distraction, which can happen as three types: (1) Drawing attention through physical actions, such as closing the eyes or ears when something frightening is seen or heard; (2) Drawing back their internal attention, such as through distraction or concentration and (3) Responding again to the attention or distraction to someone done by other people.

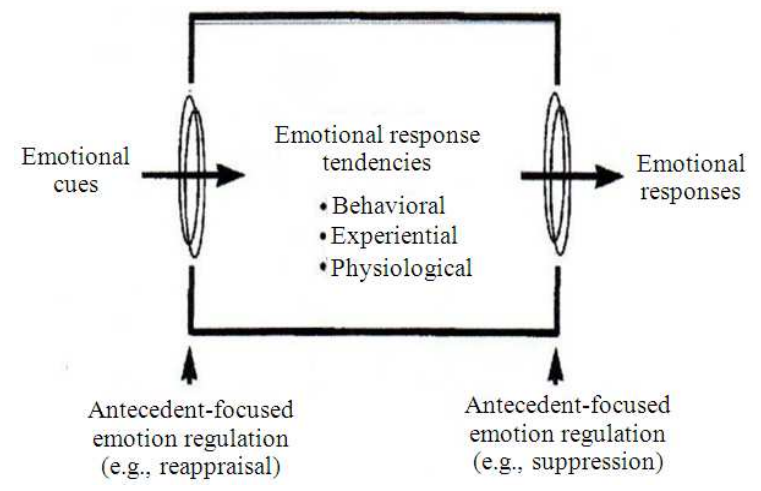

Fig. 1: A consensual process model of emotion that highlights two major classes of emotion regulation (Gross, 1998)
Cognitive change: This is the way someone changes his evaluation of a situation to change its emotion significance; it can be said to be an internal version of situation modification.

Response modulation: This is influencing emotion response (behavior, subjective experience, physiological) that has appeared. For example, expressing anger to someone or crying when feeling sad.

Based on Gross (2006) theory of emotion regulation strategy model, the following are included in the antecedent focus emotion regulation group: situation selection; situation modification; attentional deployment and cognitive change. Response modulation is included in response focus emotion regulation. Antecedent-focused emotion regulation is the regulation for manipulating input from the emotion system, so that someone is able to anticipate and regulate before an emotion appears (Fig. 1). Meanwhile, response-focused regulation is a way to regulate by manipulating outputs from the emotion system; thus regulation is done after an emotion appears.

Children with LLA need a relatively long time for the healing process. The main medical treatment is chemotherapy that generally consists of an induction phase, consolidation and maintenance that last for 2-3 years (National Cancer Institute, 2008). During this time, children are regularly and frequently hospitalized and are also out-patients. As well as chemotherapy often fails to prevent disease recurrence (Barrett and Blanc, 2009). This condition has potential to cause emotional responses. Rieffe in Vingerhoets et al. (2008) states that children with pain have more problems with emotion experiences because their internal focus is towards the disorder inside their body.

\section{MATERIALS AND METHODS}

This research uses a descriptive-qualitative method. The number of participants was 4 children aged 10 to 13 years old who had been diagnosed ALL and were undergoing chemotherapy in one of two hospitals ( $\mathrm{AB}$ and $\mathrm{XY}$ ). Two participants were survivors in early adulthood who had been considered cured from ALL. Data collection was done through semi-structured interviews with participants and parents. In addition observation was conducted to see the clinical manifestation in the participants.

The interview results were transcribed and then reread and re-discussed with the participants. Then the data was classified based on the 5 emotion regulations 
based on Gross (2006) theory for all participants through a matrix. In the matrix, 5 types of emotional regulation were observed in various phases of incidents experienced by the participants. These were diagnosis, preparation for medical treatment and medical treatment that consisted of an induction phase, consolidation or prophylaxis and maintenance.

\section{RESULTS}

Situation selection: All participants tried to reduce their sadness and fright by being physically closer to their parents, especially the mother, either when the emotion had appeared or not. They conveyed what they were feeling and asked for help to overcome that emotion. For example, they said: "When I got Leukemia, I was very sad and so I told my mom because I became calm afterwards. My mom usually finds a way to cure me". Another child said:

"When I am hospitalized like this, I want my mom to accompany me, not anyone else".

Daughters said: "It is good when mom accompanies me so when I am sad, I can talk heart to heart with her, it will be different to talk to dad".

Besides being close to the mother, the situation selection type done is by going to the hospital playground. "When I'm sad, I just play game in the playground. There are many friends there.

Situation modification: At the time they were hospitalized over a very long period for chemotherapy, all participants became bored and upset about staying in the hospital. The different environment in the hospital influenced their emotional situation. Two participants who were hospitalized at the same hospital said they were really bored with the situation in the treatment ward. One of them said: "I have been hospitalized for 3 months... ughh I feel so bored to keep staying in hospital. I don't feel free".

Other participant said: "I'm happy if there is playing activity in the hospital, but still I get bored because here is not as free as at my own home". "I just bring books to the hospital so that I don't get bored".

The situation modification that they generally did was to bring their favorite items and do fun activities. Other than that, an environmental setting such as one with fragrant rooms and without drug odors also reduced children's fright, as stated by a participant: "If the hospital smells good like this and it's no drug odor like in most hospitals, I'm happy". "I'm happy if there is a television in the hospital, so I won't get bored".
Playgrounds in the hospitals can be factor that reduces negative emotions, but these need adjustment to the children's ages. One participant said: "Playgrounds in the hospital should not only be for children, I want to play, too". Situation modification not only concerns the physical environment, it comes also from the friendly atmosphere created by hospital staff, such as doctors, nurses and volunteers. A cured survivor said: "When I was hospitalized, I felt like home, because the doctors and nurses are friendly to us". "Doctors love saying 'Hi' and asking about small things to me, such as a friendly chit chat and indeed it was not so important and not related to disease, but it was fun, we felt comfortable..and... well... I felt like at home".

A female who experienced hair loss felt embarrassed with the condition: to overcome it she wore the jilbab (veil). "I feel embarrassed with this bald head, so to overcome that feeling, I wear veil".

Situation modification done by the children to overcome their emotion was included in the physical environment aspect. In this case it was on the children's external side.

Attentional deployment: This strategy was used by many of the participants, although situation modification can also be used in other situations. For example, most participants closed their eyes when invasive action, like infuse, IT, or BMP was conducted. Indeed, other attentional deployment seems to be influenced by external agents, especially the mother. All participants said that when painful medical treatment was going to be conducted, the frightening feeling did not appear anymore or at least was reduced by distracting attention to the idea. This action was conducted to accelerate the cure. This took place mainly after participants went on medication and treatment for about one to three months. During this time parents gave participants intensive psychological support. Furthermore, parents always strengthened this process by directing participants' attention away from worries about their future by providing various solutions. One participant worried about the future because of his illness which he saw as an obstacle seen to getting a job. Parents gave information about alternative jobs that could probably be done. Participants played games or wrote to friends to overcome their sadness when they were in the hospital.

Another participant was diagnosed within 2 months and received medical treatment. He distracted his attention when going to be infused by looking in opposite directions or watching the TV available in the room. Generally, he willed himself to get well and attended the final test for elementary school students to 
enter his favored junior high school. A participant reduced his fear by doing a final test in the hospital. The participants also tried to be less sad by doing activities and not staying in bed and focusing on getting well. Another participant learned English once a week and read material about Leukemia given by the doctors but not really understanding it. Meanwhile another participant kept learning his school lessons because he wanted to get good achievements at school. With ongoing treatment over quite a long time, participants generally did not think of their illness. This was a way to distract their attention from their negative emotions.

Cognitive change: During their middle childhood, children start to be able to do emotion regulation by means of changing their thoughts. This is mainly seen at the time they have received treatment for 1-3 months. They interact with parents and communicate so that they can change their way of thinking about their illness. The following comments were made:

- The chemotherapy effect is not pleasant, but I want to be cured. To avoid sadness, I usually consider that I am still lucky compared to other patients

- I used to be afraid when I got hospitalized for the first time, but soon as I knew that many other patients who experience the same thing with me, I became more relaxed

- If I am sad all the time, I my illness will get worst.

- When I see other patient die, I am afraid, but later on I think death depends on our own body

- The Chemotherapy effect is very painful, but I think it is a way to get cured

- I think this illness is like other illness generally, so I feel confident with this condition and I feel optimistic that I can get cured

- In the beginning of the treatment, participants generally felt strong negative emotion. They still focused on their illness. A participant used to feel desperate:

- Before I knew Leukemia, I got medical treatment everywhere and it was not clear what kind of illness I suffered, doctors did not know about it yet. At the time I nearly got desperate and thought that it was ok if I died because I had no sin yet in fact I could take parents to heaven

- Other participant said:

- Sometimes when I get chemotherapy for a long time, I feel desperate, too, but I remember what my mother told me". "How come you would give up after struggling so far? Giving up means stop living
Response modulation: In all the existing cases, response modulation occurred mainly at the beginning of Leukemia diagnosis, when children were still shocked. They did not understand and did not believe what was happening to them and they received treatment procedures that were not always successful, or had unavoidable therapy effects. However, as time went by, participants generally coped better by doing regulation through response modulation.

At the time I was hospitalized. I wanted to go home all the time. I remained silent and did nothing in the hospital.

Female participants generally cried when they felt sad or afraid:

- In the beginning of being hospitalized, I kept crying in the hospital and I was afraid that I could not be cured

- If I am sad, I will cry

- Cultural aspects influenced emotional response in some. Individuals in a collective culture will place existing social harmony as a priority. Two participants said:

- Chemo medicine used to make my skin burnt, I was angry but I could only cry at the time, if I got angry at the hospital staff, then I would be considered impolite

- A doctor used to make mistakes in conducting BMP, I was upset and did not want to meet the doctors at the time

\section{DISCUSSION}

Based on the emotion regulation concept of Holodynski and Fridelmeier (2006), human growth is based on an internalization process. The older they are, their emotion regulation will be more intrapersonal. It means that an individual is able to independently undertake emotion regulation. During the period of adolescence, Holodynski and Fridelmeier stated that individuals are able to do emotion regulation intrapersonally while the interpersonal role is decreasing. However, most participants, especially in the beginning of diagnosis of Leukemia, still show high interpersonal regulation. This is especially in situation modification, in which the role of parents, especially the mother, is so significant in forming the emotion regulation of children. As participants get medical treatment, they begin to use intra-personal emotion regulation. According to Thompson (1994), children and adults generally seem to be able to regulate emotion if they can find a way to express it adaptively instead of maladaptively. Adaptive response alternatives provide an 
adaptive process that enables children to learn how to select emotion regulation that can make them comfortable and capable of adapting (Gross, 2006). The ability to select alternatives by the participants themselves and using personal responsibility enables children to regulate emotion.

Cultural factors can be global factors. Kitayama et al. (2004) and Matsumoto et al. (2008) claim that Eastern culture that is based on collectivity tends to form engagement towards social support when someone experiences emotion. On the other hand, Western culture tends to be individualistic. It will tend to form disengagement from social support, in the case where someone uses intra-personal regulation.

Cultural aspect also affect motivation factors and norms where either Western or Eastern culture promotes emotion regulation behavior on the basis of maintaining social harmony. However, Kitayama notes that interpersonal relationships in Eastern culture are stronger in keeping this social harmony if someone experiences negative emotions towards other people who will probably hurts him, he will repress the emotion (suppressive). Thus social motivation can promote how emotion regulation is conducted. According to Philippot and Feldman (2004) people's motivation tend to demand comfortable situations so that they avoid negative situations in terms of interpersonal relationships.

The hospital environment as a factor seems to play an important role as a situation modification strategy by participants during ongoing medical treatment in the hospital. A comfortable hospital situation which is not frightening, but makes children feel at home, is a factor that will increase emotion regulation. The children's psychological situation is that they face a series of chemotherapy and medical treatments that will be painful. The children have to face this willingly or not, so the psychological situation can be modified by a friendly hospital environment. The $\mathrm{AB}$ hospital has a motto in the children's rooms: "No scared no pain". This supports the participants' process of situation modification, which can also be a psychological distraction. Good room facilities, good communication between children, family, hospital staff and volunteers help provide this environment. This can also increase children's emotion regulation through attentional deployment in the form of distraction through external assistance for children. According to Skeen (2007), talking to children and listening to children's complaints that are undergoing long treatment because of serious illness like cancer is beneficial. This can be psychological therapy where adults can know how children feel subjectively. Holodynski and Fridelmeier
(2006) mention too that developing children's verbal capability is a means of directing them to be able to undertake intra-personal emotion regulation.

This research found that female participants seemed to show embarrassment related to hair loss and baldness, whereas male participants do not. The gender aspect plays a role in the carrying out emotion regulation (Garnefski et al., 2007). Females are considered to do more rumination or thinking too much about negative things. Females also go through catastrophizing, in which they feel that an incident where they experienced showing emotion was frequently considered a bad experience. The hair lost to baldness is probably considered as a negative thing that threatens their physical appearance. Probably, the value of appearance is different for females and males.

Emotion regulation in children demands very much external support. Besides support from their nannies, physical situation support, especially the hospital setting, also plays an important role in supporting children's emotion regulation. Settings with hospital wards that are not 'frightening' and the existence of playgrounds appropriate to their level of development are very helpful. They can distract children and such situation modification can reduce negative emotion and this helps create positive emotion. Other than that, children should be given opportunities to reveal and verbally express what they know, what they feel and what they hope. These are the basic abilities for regulating emotion in the sense of internally cognitive changes. Through discussion with children, external assistance can be a symbolic strategy (symbolic strategic/cognitive reinterpretation). Children starting from aged 3-6 years old have been able to do selfinstruction about what they have done; in this case, emotion regulation (Holodynski and Fridelmeier, 2006).

\section{CONCLUSION}

In facing negative emotion in the beginning of diagnose period, children do more emotion regulation. They focus on responses (response focus), they repress their feelings (suppresive), they express behavior such as crying and they reject medical staff and refuse medical treatment. They also express their feelings verbally. Meanwhile, chemotherapy may be nearly complete and the induction period taking place. External assistance is given to children by an outside party and children start to try causing distractions. They accept emotion regulation focusing on antecedents (antecedent focus) in the form of situation selection, modification selection, attentional deployment and cognitive change. 
During the consolidation period or the prophylaxis of the chemotherapy process, children are becoming able to independently undertake emotion regulation that focuses on antecedents. In the prior period of chemotherapy, children mainly practice emotion regulation interpersonally.

Emotion, along with its regulation, is a process of repeating that can be continuous and dynamic that can be described as a feedback loop. This situation can create certain new responses and so on. Through the repeated situation-response process, children with their capacity for development and supported by external stimulus are able to select certain adaptive responses. These are well known as adaptive response alternatives and are applicable in various conditions.

Through adaptive response alternatives processes, children are able to select alternative emotion regulation and they can be adaptive during the process of medical treatment. Thus there will be changes through gradual process in various dynamic situations and emotion internal emotion regulation transforming to intrapersonal emotion regulation.

The transformation of emotion regulation to the intrapersonal direction during an individual's process of medical treatment can last until that person is declared cured. This can be seen in the survivors who have been through the period of treatment and have been declared cured. Because of these conditions, a situation faced during the process of treatment can be an opportunity for internalizing the process of emotion regulation in children when children have gone through the medical treatment. The children will be able to apply emotion regulation adaptively in various situations.

In addition to the children's internal ability, these changes need supportive roles to be played by external parties. These may be parents (this research focused on mothers), hospital personnel, (doctors, nurses, psychologists, volunteers, other patients, friends), hospital facilities, hospital policies in the form of service policy and good educational opportunities and job prospects.

Implications for clinical practice and further research: Hospitals and medical staff should assist parents to fit in the time to accompany their children. Processes should be put in place to supporting children's emotion regulation. Services should be prepared to support aspects of education for children and to organize volunteers for accompanying children.

Research could be conducted to see the influence of emotion regulation internalized as part of daily functions as soon as the treatment period is finished (post treatment). For example, by comparing emotion regulation of children at the start of medical treatment to when treatment is ongoing and when it is finished.

Further research could be conducted focusing on individual's experiences that have been declared totally cured of Leukemia. This could be done through phenomenological research observing various aspects such as the influence of the family dynamic and culture.

\section{REFFERENCES}

Andersen, B.L., D.M. Golden-Kreutz and V. Dilillo, 2000. Cancer. In: Handbook of Health Psychology, Baum, A.S., T.A. Revenson and J.E. Singer (Eds.). Psychology Press, Erlbaum, Mahwah, NJ., pp: 709725. ISBN-10: 0805814957

Barrett, J. and K.L. Blanc, 2009. Cancer chemotherapy and immune regulation. Am. J. Immunol., 5: 8-16.

Chen, E., L.K. Zeltzer, M.G. Craske and E.R. Katz, 2000. Children's memories for painful cancer treatment procedures: Implications for distress. Child Dev., 71: 933-947. DOI: 10.1111/14678624.00200

Cole, P.M. and B.L. Tamang, 1998. Nepali children's ideas about emotional displays in hypothetical challenges. Dev. Psychol., 34: 640-646. PMID: 9681255

Cole, P.M., C.J. Bruschi and B.L. Tamang, 2002. Cultural differences in children's emotional reactions to difficult situation. Child Dev., 73: 983996. DOI: 10.1111/1467-8624.00451

Cole, P.M., S.E. Martin and T.A. Dennis, 2004. Emotion regulation as a scientific construct: Challenges and directions for child development research. Child Dev., 75: 317-333. PMID: 15056186

Coniglio, S.J., 1995. Developmental outcome of childhood leukemia. Topics Early Cildhood Special Educ., 15: 19-31. DOI: 10.1177/027112149501500104

Garnefski, N., F. Jellesma, M.M. Terwogt and V. Kraij, 2007. Cognitive emotion regulation strategies and emotional problems in 9-11 year old children. Eur. Child Adolesc. Psychiatry, 16: 1-9. PMID: 16791542

Gross, J.J., 1998. Antecedent-and respon-focused emotion regulation: Divergent consequences for experience, expression and physiology. J. Personality Soc. Psychol., 74: 224-237. PMID: 9457784

Gross, J.J., 2006. Handbook of Emotion Regulation. 1st Edn., The Guilford Press, New York, ISBN-10: 1593851480, pp: 654. 
Holodynski, M. and W. Fridelmeier, 2006. Development of Emotions and Their Regulation. Birkhauser, New York, ISBN: 0387232818, pp: 265.

Kazak, A.E., 1994. Implications of Survival: Pediatric Oncology Patients and Their Families. In: Pediatric Psycho-Onchology: Psychological Perspectives on Children with Cancer. Bearison, D.J. and R.K. Mulhern (Eds.). Oxford University Press, New York, ISBN: 0195079310, pp: 247-247.

Kitayama, S., M. Karasawa and B. Mesquita, 2004. Collective and Personal Proceeds in regulating Emotions: Emotion and Self in Japan and the United States. Dalam The Regulation of Emotion, Lawrence Erlbaum Associates Publishers, New Jersey, ISBN: 0805842012, pp: 251-273.

Kreitler, S. and M.W.B. Arush, 2004. Psychosocial Aspects of Pediatric Oncology. 1st Edn., John Wiley and Sons, Ltd., Sussex, ISBN-10: 0471499390, pp: 470.

Kupst, M.J., M.B. Natta, C.C. Richardson, J.L. Schulman and J.V. Lavigne et al., 1995. Family coping with Pediatric Leukemia: Ten years after treatment. J. Pediatr. Psychol., 20: 601-617. PMID: 7500233

Matsumoto, D., S.H. Yoo, S. Nakagawa, 2008. Culture, emotion regulation and adjustment. J. Pers. Soc. Psychol.. 94: 925-937. PMID: 18505309

Middendorp, H.V., R. Geenen, M.J. Sorbi, J.J. Hox and A.J. Vingerhoets et al., 2005. Styles of emotion regulation and their associations with perceiveed health in patients with rheumatoid arthritis. Soc. Behav. Med., 30: 44-53. PMID: 16097905

National Cancer Institute, 2008. General information about childhood Acute Lymphoblastic Leukemia. US National Institute of Health.

Philippot, P. and R.S. Feldman, 2004. The Regulation of Emotion. 1st Edn., Psychology Press, New Jersey, ISBN-10: 0805842012, pp: 432.
Sarafino, E.P., 2005. Health Psychology Biopsychosocial Interactions. 5th Edn., John Wiley and Sons, Inc., New Jersey, ISBN-10: 0471691003, pp: 568.

Scwartz, L. and D. Drotar, 2006. Posttraumatic stress and impairment in survivors of childhood cancer survivors in early adulthood compared to healthy peers. J. Pediatr. Psychol., 31: 356-366. DOI: 10.1093/jpepsy/jsj018

Speechley, K.N., M. Barrera, A.K. Shaw, H.I. Morrison and E. Mansell, 2006. Health related quality of life among children and adolescent survivors of childhood cancer. J. Clin. Oncol., 24: 2536-2543. DOI: $10.1200 /$ JCO.2005.03.9628

Thompson, R.A., 1994. A theme in search of definition. Monogr. Soc. Res. Child Dev., 59: 25-52. PMID: 7984164

Vingerhoets, A., Nyklicek and M. Zeelenberg, 2008. Emotion Regulation: Conceptual and Clinical Issues. Springer Science+Bussiness Media, New York, ISBN-10: 0387299858, pp: 256.

Zebrack, B.J., L.K. Zeltzer, J. Whitton, A.C. Martens and L. Odom et al., 2002. Psychological Outcomes in longterm survivors of childhood leukemia, Hodgkin's disease and non-Hodgkin's lymphoma: A report from the childhood cancer survivor study. Pediatrics, 110: 42-52. PMID: 12093945

Zeltzer, L.K., E. Chen, R. Wess, M.D. Guo and L.L. Robison et al., 1997. Comparison of Psychologic outcome in adult survivors of childhood acute lymphoblastic leukemia versus sibling controls: A cooperative children's cancer group and National Institutes of Health Study. J. Clin. Oncol., 45: 547556. PMID: 9053476 